\title{
Isquemia mesentérica: manejo endovascular
}

\author{
Mesenteric ischaemia: the endovascular management

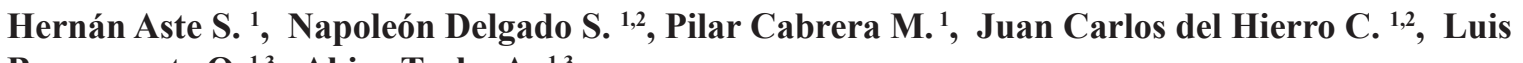 \\ Bracamonte O. ${ }^{1,3}$, Akira Tsuha A. ${ }^{1,3}$
}

\section{RESUMEN}

La isquemia mesentérica es una condición que puede volverse muy grave y ser letal, más aún en los casos agudos. Un tratamiento precoz es muy importante. Se presentaron cuatro casos consecutivos tratados por nuestro equipo con trombosis, o disección o aneurismas de arterias mesentérica superior y tronco celíaco, se revisaron las indicaciones y se evaluaron los tratamientos llevados a cabo. Se utilizaron procedimientos endovasculares en todos, técnicamente en forma exitosa, evitando que progrese a un desenlace peligroso. Hubo diversidad de técnicas endovasculares adaptándolas a cada caso. En ningún paciente se requirió un acceso quirúrgico abierto para completar el tratamiento realizando las intervenciones prontas. El manejo endovascular de las lesiones mesentéricas es factible y replicable, y en nuestros casos podría haber prevenido una necrosis intestinal así como la necesidad de cirugías resectivas.

PALABRAS CLAVE: Isquemia mesentérica, procedimientos endovasculares, trombosis. (Fuente: DeCS BIREME)

\section{SUMMARY}

Mesenteric ischaemia is a condition that may be very severe and potentially lethal particularly in the acute presentation. Early treatment is crucial. We present four consecutive cases managed by our team with thrombosis or dissection or aneurysm of the superior mesenteric artery and the celiac trunk reviewing indications and treatment implemented. Multiple successful endovascular procedures were used in all cases avoiding progression to lethal consequences. None of the patients required an open surgical procedure. Therefore, endovascular procedures are feasible in these patients avoiding intestinal necrosis as well as the need for open surgeries.

KEYWORDS: Mesenteric ischemia, endovascular procedure, thrombosis. (Source: MeSH NLM).

\section{INTRODUCCIÓN}

La isquemia mesentérica aguda, a diferencia de la crónica, es una entidad, poco frecuente, pero de elevada mortalidad, y esta mortalidad está relacionada al retraso en el diagnóstico y tratamiento, siendo mayor de $60 \%$ si el retraso es mayor de 12 horas, y entre 80 y $100 \%$ si es de 24 horas o más ${ }^{(1)}$.
Se presenta en uno a dos casos por cada 1000 admisiones por año, a pesar de que en necropsias no seleccionadas se encuentra ateroesclerosis mesentérica en 35 a $70 \%$ de $\operatorname{casos}^{(2)}$.

La isquemia mesentérica aguda puede ser de origen arterial (trombótico, embólico o por disección), de origen venoso (trombótico) y de causas no oclusivas

\footnotetext{
Clínica SANNA. San Borja, Lima, Perú.

Clínica Delgado. Lima, Perú.

Clínica Ricardo Palma. Lima, Perú.
} 
(hipotensión, bajo débito, farmacológico, etc). La disección es una entidad poco frecuente ${ }^{(3)}$.

La definición de isquemia mesentérica aguda es, según Motta: "Entidad clínica multicausal producida por la interrupción brusca del aporte sanguíneo a un determinado segmento intestinal, lesionándolo inicialmente de forma reversible, pero que si se mantiene el tiempo suficiente, hace que deje de ser viable y evolucione a necrosis completa de su pared, asociado entonces a elevada mortalidad" (1).

Por otro lado, tenemos la isquemia mesentérica crónica, cuya incidencia exacta no se conoce bien, pero se estima que es uno de cada 100,000 admisiones por año ${ }^{(2)}$; y suele comprometer dos o más arterias viscerales, y puede llevar a la caquexia al paciente, pero cuando se reagudiza se vuelve muy riesgosa, y tiene origen ateroesclerótico generalmente.

El manejo de la isquemia intestinal aguda puede ser médico o quirúrgico, y cuando es quirúrgico puede ser endovascular o por cirugía abierta, y la cirugía abierta puede ser resectiva o terapéutica.

Se presentan cuatro casos tratados con manejo endovascular, de casos en etapa aguda, se evaluaron las indicaciones, el tratamiento y las complicaciones, además de una revisión de la literatura respecto a esta patología.

\section{PRESENTACIÓN DE LOS CASOS}

\section{Caso 1}

Varón de 56 años, con antecedente de hipertensión arterial, que acudió por emergencia de nuestro centro con un tiempo de enfermedad menor de dos horas, caracterizado por dolor abdominal intenso, asociado a náuseas y vómitos. Además, deposiciones oscuras y líquidas. Al examen clínico no se encontraron signos de peritonismo, ni signos de alarma abdominal, pero sí taquicardia leve, hipertensión arterial, palidez y diaforesis. Se solicitó análisis de laboratorio y se encontró hemoglobina en $18 \mathrm{gr} / \mathrm{dl}$, glicemia en $186 \mathrm{mg} / \mathrm{dl}$, y leucocitos en sangre dentro de valores normales sin presencia de elementos jóvenes. Se evaluó la función coronaria sin encontrar signos patológicos. Ante la intensidad del dolor se solicitó una angio tomografía de aorta en la sospecha de posible disección de esta arteria. El resultado fue: "Disección espontánea de la capa íntima de la Arteria Mesentérica Superior (AMS), con trombosis evolutiva a nivel de su luz falsa, que presenta dilatación sacular distal y obturación parcial de la luz verdadera en aproximadamente 95\% [tipo IIb de Yun] (4). Asoció extensión trombótica hacia las ramas arteriales ileales [tipo D de Luan] (5) y signos de incipiente mesenteritis de distribución perivascular" (figura 1 (A)).
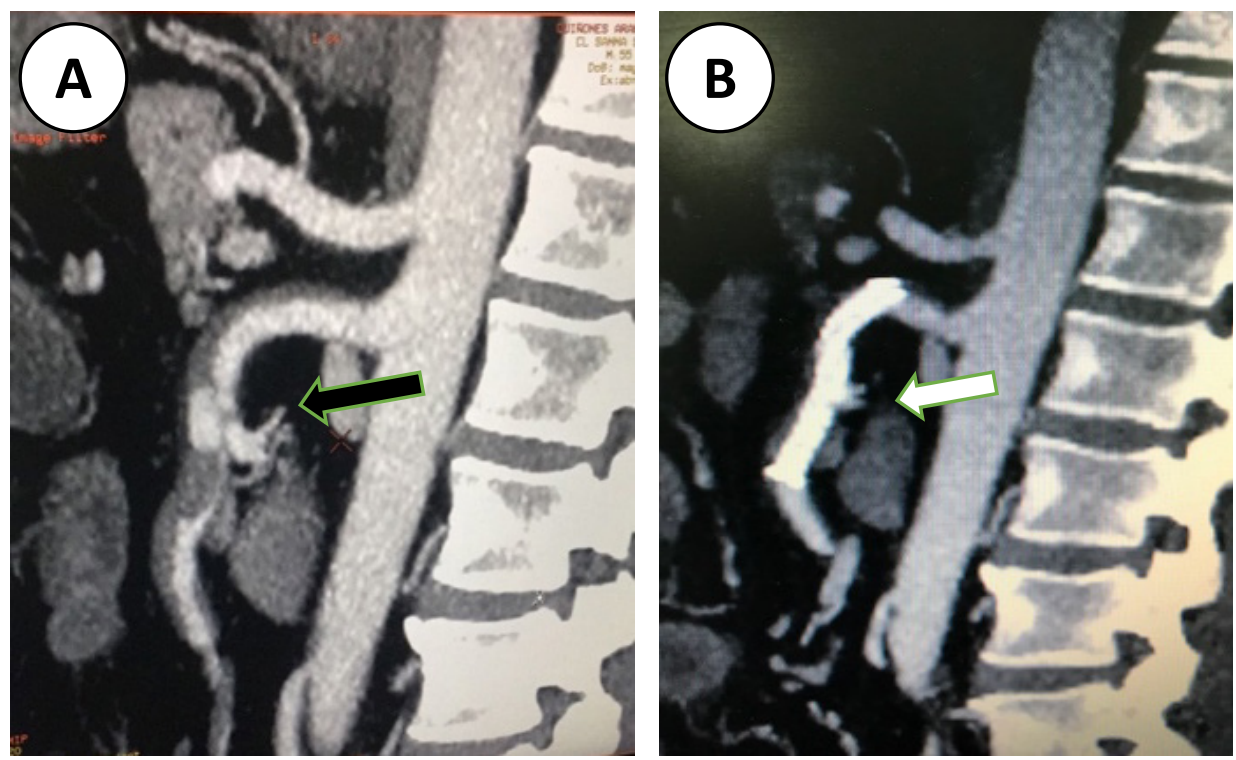

Figura 1. A: Se muestra la arteria mesentérica superior con disección y trombosis de luz falsa. Rama colateral saliendo del medio de la disección (flecha negra). B:

Control al mes post operatorio demostrando patencia de los stents en AMS y de la rama colateral (flecha blanca). 
Dado el cuadro clínico agudo y lo incipiente de los signos vasculares, se decidió revascularizar y tratar la disección de la arteria mesentérica superior por técnicas endovasculares.

Se realizaron las evaluaciones y medidas de las imágenes tomográficas en un aplicativo de imágenes digital, que permitió tener una valoración tridimensional de los vasos estudiados. En el aplicativo se pudo obtener la medida del vaso, las ramas colaterales, y la indicación y tamaño de los dos stents metálicos abiertos que requería el caso.

Se ingresó por accesos braquial izquierdo y femoral derecho con introductores largos hasta la aorta abdominal donde se canalizó el ostium de la arteria mesentérica superior con la colocación de una guía hidrofílica 0,035 ". Previamente se "heparinizó" manteniendo un tiempo de coagulación activado (ACT) mayor de 400". Se realizó una angiografía de control para confirmar los hallazgos tomográficos.

Se procedió a cambiar de guía por una de mayor solidez y a implantar el primer stent distal abierto de 6 × $60 \mathrm{~mm}$, para luego desplegar el segundo stent similar de $8 \times 60 \mathrm{~mm}$. Se completó el procedimiento "balonando" el proximal con un balón de 8 x 40 $\mathrm{mm}$. Se confirmó la presencia de una rama colateral patente que salía en medio de la disección. Se procedió al retiro de introductores y cierre de orificios de punción con sutura vascular percutánea.

La evaluación post operatoria fue favorable, con disminución marcada del dolor. No fue necesaria exploración quirúrgica de otro tipo. El alta fue a los 10 días de la operación.
Al mes del procedimiento, se realizó una angiotomografía encontrando patencia de la arteria mesentérica superior y por lo tanto de los stents, y además de la rama colateral, con buena perfusión distal (figura 1 (B)).

\section{Caso 2}

Varón de 36 años, que acudió por emergencia con antecedentes de hipertensión arterial, sobrepeso y registro de haber sido sometido a colocación de dos stents en arteria mesentérica superior 3 años antes en otro centro médico.

Ingresó presentando dolor tóraco-abdominal de cuatro días de evolución que se fue haciendo intenso 8/10 (AVA), que por momentos empeoraba, llegando a emergencia con dos horas de agravamiento. Al examen se encontraba hemodinámicamente estable, con abdomen blando, depresible, poco expresivo.

Por las características del dolor se solicitó la determinación de enzimas cardiacas (CPK-MB y troponina), asimismo enzimas pancreáticas (lipasa y amilasa), cuyos resultados fueron normales. Leucocitos en sangre resultó en 7000 por $\mathrm{mm}^{3}$ con bastones en $0 \%$; lactato sérico fue $2,6 \mathrm{mmol} / 1(\mathrm{VN}$ : $\leq$ $1,8)$.

Por los antecedentes y el dolor se solicitó una angiotomografía de aorta descendente, encontrándose presencia de disección del origen del tronco celiaco (TC) y patencia de los stents mesentéricos, aunque el más distal estaba adelgazado $(3,5 \mathrm{~mm})$, y una dilatación aneurismática en el segmento de arteria entre dos dispositivos; el stent proximal medía $6 \mathrm{~mm}$ (figura 2).

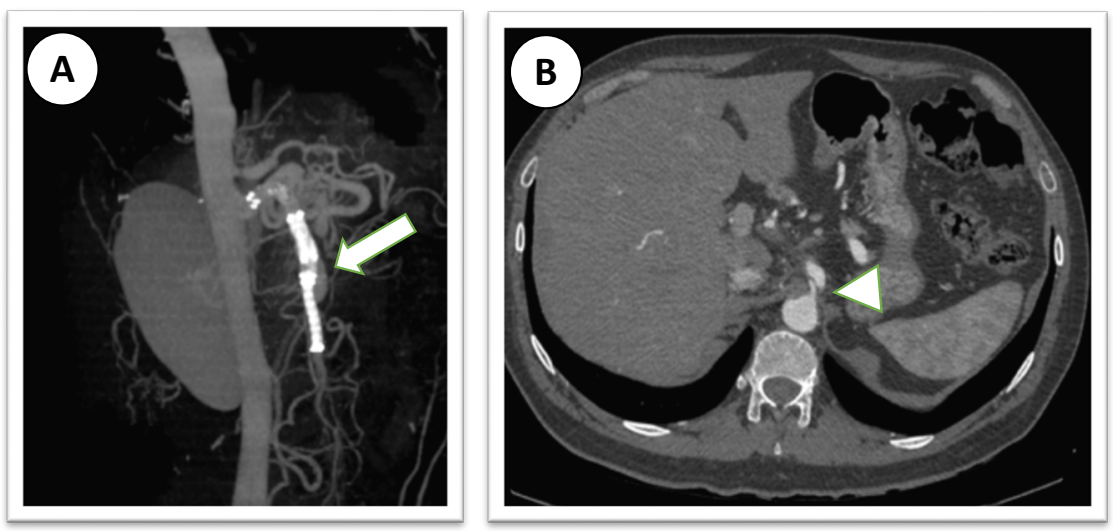

Figura 2. A: Aneurisma de arteria mesentérica superior entre stents (Flecha blanca). B: Disección de origen de tronco celiaco (Cabeza de flecha). 
El problema surgió en definir cuál de los hallazgos era la causa del dolor, por lo que se decidió tratar ambas arterias.

Mediante acceso desde ambas femorales y utilizando introductores largos se canalizó inicialmente el ostium de la arteria mesentérica superior, y se realizó una angiografía de base. Se descubrió un tercer stent abierto en el proximal. Se procedió a colocar un stent recubierto de $58 \times 5 \mathrm{~mm}$ entre ambos stents previos, con una superposición en ambos. Dada la diferencia de calibres entre ambos stents previos se decidió dilatar el segmento proximal del stent recubierto, con un balón de 8 x $40 \mathrm{~mm}$ con lo que se logró igualar el diámetro proximal cubriendo totalmente la zona aneurismática. Posteriormente se accedió al tronco celiaco con guía hidrofílica y se evaluó la primera rama que aparecía a $2,8 \mathrm{~mm}$ del origen. Se colocó un stent recubierto de $37 \times 7 \mathrm{~mm}$, dejando unos milímetros dentro de aorta abdominal para no cubrir la primera rama.

El control angiográfico mostró patencia de ambas arterias y ausencia de dilatación aneurismática y de disección. Se procedió al cierre de ambos accesos con sutura percutánea.

La evolución post operatoria fue adecuada con disminución importante del dolor, saliendo de alta a los cuatro días después de la cirugía.

En el seguimiento, un mes después de su alta reingresó por emergencia por presentar melena, cansancio fácil, palidez y nauseas. Se pensó en sangrado por el uso de antiagregantes plaquetarios, pero la endoscopía baja encontró una úlcera con vaso visible en la válvula ileocecal; se procedió a escleroterapia + clip.

\section{Caso 3}

Mujer de 79 años. Acudió a nuestro centro por dolor abdominal difuso, malestar general, escalofríos, y dolor en cadera izquierda. Además presentó alza térmica, deposiciones líquidas sin moco ni sangre. Al examen, el abdomen era blando y los ruidos hidroaéreos estaban aumentados. El dolor era persistente, difuso y de mediana intensidad. Se le realizaron evaluaciones diversas durante una semana, entre ellas una angiotomografía de aorta abdominal para descartar isquemia mesentérica. Los análisis de laboratorio mostraron leucocitos en $10200 \mathrm{x} \mathrm{mm}^{3} \sin$ bastones; leucocituria y proteína $\mathrm{C}$ reactiva en 186.

La angiotomografía realizada una semana de su ingreso, mostró trombosis del origen de arteria mesentérica superior mayor al $60 \%$, con la primera rama naciendo a $4,5 \mathrm{~cm}$ del origen (figura 3 ). No hubo signos que indiquen alteración de perfusión intestinal.

La planificación digital sugirió acceso braquial izquierdo y femoral derecha, para colocar un stent recubierto. Con esas indicaciones se procedió a canular desde el brazo, el ostium de arteria mesentérica superior, se canalizó con guía y catéter, y se colocó y

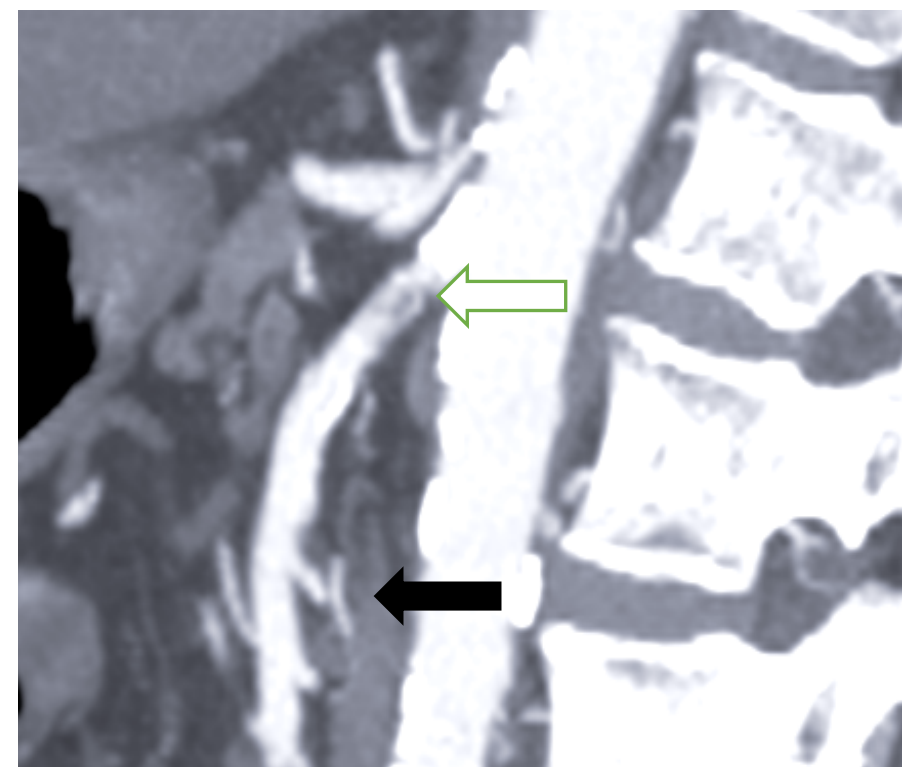

Figura 3. Trombosis de origen de AMS (flecha blanca). Primera rama a 4,5 $\mathrm{cm}$ de origen (flecha negra). 


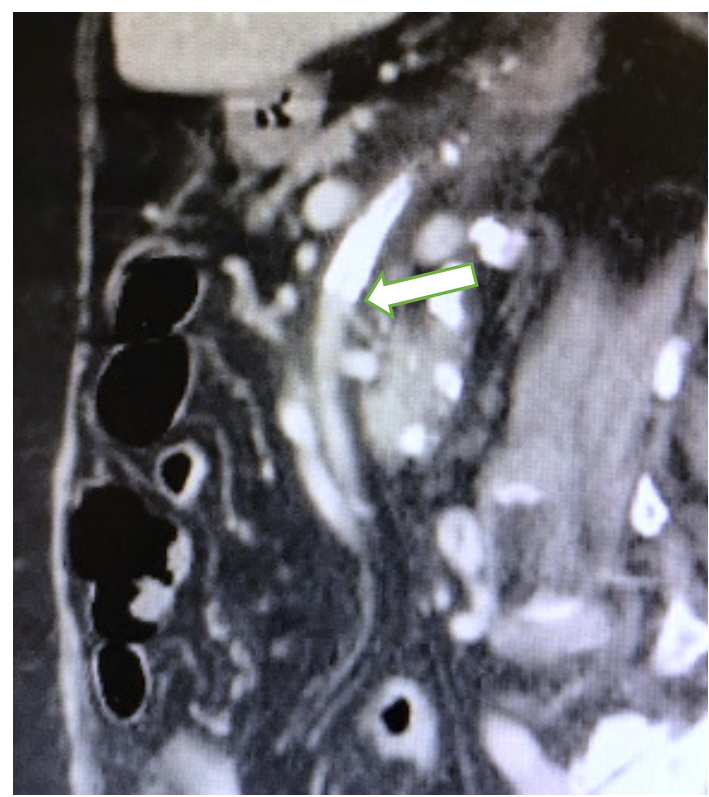

Figura 4. AMS con stent cubierto patente (flecha blanca).

desplegó un stent recubierto de 8 x $57 \mathrm{~mm}$, dejando unos milímetros en la aorta para no ocluir la primera rama. El procedimiento se realizó sin complicaciones y se efectuó el cierre con sutura percutánea.

La evolución de la paciente fue tórpida, presentando dolor abdominal difuso que cconllevó a reingresos posteriores; sin embargo, tanto en ecografías como en el control angio tomográfico mostraron patencia de la AMS sin trombosis y manteniendo patente la primera rama (figura 4), inclusive la endoscopía alta y baja mostró cicatriz de úlcera duodenal, y mucosas bien perfundidas. Se catalogó el dolor como de origen neuropático asociado a una paniculitis de pared.

\section{Caso 4}

Mujer de 89 años, con antecedente de hipertensión arterial, hipotiroidismo y fibrilación auricular controlada; ingresó por emergencia por dolor intenso de 48 horas de evolución, tipo hincada, con irradiación dorso lumbar, acompañado de náuseas y vómitos alimenticios y luego salival. Se añadió fiebre de $38^{\circ} \mathrm{C}$. Al examen se encontró Glasgow 14/15 y punto de Murphy (+).

Mediante ecografía abdominal al ingreso se diagnosticó colecistitis aguda y fue sometida el mismo día, a colecistostomía de drenaje debido a que su estado general no era adecuado para extirpar la vesícula. Con ello la paciente mejoró, pero persistió con el dolor y su estado general no era apropiado.
A semana de la cirugía, se le realizó una tomografía la que sugería una lesión de arteria mesentérica superior detectándose un trombo oclusivo desde el origen de ésta, y sin signos de alarma intestinal. No se pudo identificar con facilidad circulación colateral que compense la trombosis. Además, se encontró derrame pleural leve a moderado en ambos hemitórax. Con esta información y debido al estado general de la paciente se decidió ingresar a sala de operaciones para intentar revascularizar dicha arteria.

Mediante acceso por ambas femorales, y luego de muchos intentos, se logró canalizar una arteria mesentérica de calibre distal muy delgado en sus ramas. Debido a lo estrecho de la lesión se tuvo que hacer una predilatación con un balón delgado de $3 \mathrm{~mm}$ para luego colocar otro balón de $6 \mathrm{~mm}$, siendo difícil abrir la lesión. Lamentablemente se encontró una rama muy cerca al origen, a menos de $4 \mathrm{~cm}$, y los stents que se disponían eran muy largos, teniendo riesgo de ocluir dicha rama, por ello se decidió realizar solo la angioplastia con balón.

La evolución fue muy buena, con mejoría de los marcadores inflamatorios y adecuada disminución del dolor. Sin embargo, a los pocos días nuevamente presentó deterioro por lo cual se solicitó una angio tomografía de control. En esta tomografía se evidenció que aún había presencia del trombo oclusivo, con relleno de la arteria por colaterales y sin signos de sufrimiento intestinal por ese método (figura 5).

La paciente no mostró signos de daño intestinal, pero sí evidencias de colecistitis reagudizada, por lo que fue reevaluada por cirugía general quienes

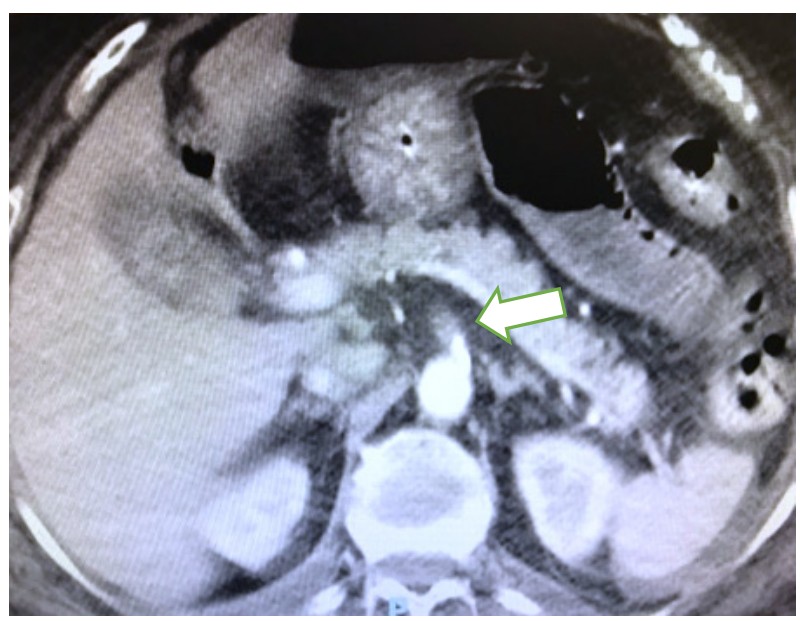

Figura 5. Trombosis de origen de AMS (Flecha blanca). 
decidieron realizar una colecistectomía. Luego de completada esta operación la paciente se recuperó notablemente; el informe del cirujano fue que las asas intestinales se encontraban bien perfundidas. La familia decidió observar la evolución antes de re intentar una colocación de stent para abrir definitivamente el vaso.

\section{DISCUSIÓN}

La isquemia mesentérica aguda es una entidad grave, y puede ser de origen arterial, venoso, o no oclusivo. La de origen arterial puede ser embólica, trombótica, disección o compresión. La de origen venoso suele ser trombótica mayormente. La no oclusiva se debe a alteraciones de flujo como bajo gasto cardiaco, hipotensión, drogas, etc. La forma más frecuente es la arterial y embólica con $50 \%$ de incidencia. La isquemia mesentérica puede ser también crónica, más frecuente en mujeres (hasta $70 \%{ }^{(6)}$, de origen ateroesclerótico; sin embargo, es la isquemia aguda o la reagudizada, la de mayor mortalidad.

La mortalidad, en casos agudos, está directamente relacionada a la demora en el diagnóstico y el tratamiento. Cuando el diagnóstico tarda más de 12 horas, la mortalidad es superior a $60 \%$, y si pasa de 24 horas, llega a 80 ó $100 \%{ }^{(1)}$. En nuestros cuatro casos hubo dos vasos con trombosis, dos vasos con disección y un caso asociado a un aneurisma entre dos stents previos en otro vaso y publicado en el año 2017 por el grupo que lo atendió en su primer evento ${ }^{(7)}$; el diagnóstico fue hecho en corto tiempo desde que llegó el paciente a la emergencia. Se presentan entonces tres casos crónicos reagudizados y un agudo.

Las disecciones, per se, son infrecuentes y se han publicado pocos casos en la literatura, 200 casos de TC y 250 de AMS ${ }^{(3,8)}$. Los factores causales de disección pueden ser alteraciones de tejido conectivo como la Displasia fibromuscular o la Medio lisis arterial segmentaria (SAM), entidad no ateroesclerótica, no inflamatoria y de causa desconocida ${ }^{(8)}$, y también se ha asociado a un efecto mecánico hidrodinámico cuyas fuerzas de fricción pudieran ser causa de disección (shear stress) ${ }^{(9)}$.

Las disecciones se pueden manejar de manera conservadora ${ }^{(3,8,9)}$, llegando a una intervención solo en casos seleccionados como persistencia de dolor, isquemia intestinal, etc. Los casos presentados en nuestra serie se trataron por persistencia del dolor y con importante área en riesgo evidenciada por la angio tomografía multicorte en los casos 1, 2 y 3 ; y el caso 4, se trató por el deterioro clínico de la paciente asumiendo que un factor causar podría haber sido la isquemia intestinal. No se pudo identificar un origen embólico claramente; se sabe que generalmente los émbolos se suelen alojar en las arterias distales, hasta el nivel de la cólica media, y sólo el 15\% es en el origen de la misma arteria mesentérica ${ }^{(1)}$. En la trombosis, en cambio, suele encontrarse dicho trombo en el origen del vaso. Nuestros dos casos de trombosis fueron en el origen del vaso (casos 3 y 4), con características de cronicidad y signos de reagudización.

Los cuatro casos tenían hipertensión arterial, a pesar de no ser necesariamente la tendencia de otras series ${ }^{(4)}$. Las edades fueron variables y el género estuvo dividido equitativamente. Sin embargo, sin ser una afirmación de tendencia, las disecciones fueron en los varones jóvenes, coincidiendo con la serie de Yun ${ }^{(4)}$, y la trombosis en las mujeres mayores. Solo se demostró fibrilación auricular en la paciente de 89 años (caso 4), en la que la trombosis fue el mecanismo patológico, sin descartar que pueda haber componente embólico con alojamiento proximal; pero la retrombosis que presentó, sugiere más un origen trombótico.

En todos se usó la angiotomografía con cortes de $1 \mathrm{~mm}$ o menos, recomendación clase I, y nivel de evidencia $\mathrm{B}{ }^{(10)}$. Por el momento, no existen marcadores de laboratorio para trombosis que confiablemente nos lleven al diagnóstico en esta patología; quizá el dímero-D sea el más cercano por su elevada sensibilidad, pero tiene en contra su baja especificidad, con un mejor valor predictivo negativo (10) (recomendación clase I, Nivel de evidencia B).

En el caso número 2, el dolor se asoció a dos lesiones en vasos contiguos, tronco celiaco disecado y arteria mesentérica tratada previamente con stents, que hizo un aneurisma entre dos de los dispositivos que no tenían sobre posición (destelescopaje). Esto hizo el manejo un poco más complicado, pues había que tratar un vaso ya manipulado, con dos patologías (aneurisma y estenosis intra stent), asociado a una disección del TC aparentemente antigua ${ }^{(7)}$. Se usó stents en tres de los cuatro casos, y en uno de ellos con más de dos dispositivos.

En la isquemia crónica, el manejo endovascular es interesante pues, comparándolo a la cirugía abierta, muestra similar patencia a corto plazo y menor morbilidad, aunque no muestra mayor sobrevida a 90 
días y 6 meses ${ }^{(2)}$. Asimismo, hay un mayor número de re-estenosis y reintervenciones comparando estudios retrospectivos con cirugía abierta ${ }^{(11)}$. En la isquemia mesentérica crónica, tratada con stents, el porcentaje de re-estenosis y reintervenciones oscila entre 20 y $66 \%$. Puede, sin embargo, ser un procedimiento que preceda a una cirugía abierta que economice la extensión de la resección intestinal.

En isquemia crónica tratada con stent, se puede detectar estenosis recurrente con eco Doppler, midiendo la velocidad de flujo cuando es $>330 \mathrm{~cm} / \mathrm{s}$, o cuando en una angiografía se encuentra $>50 \%$ de estrechez luminal ${ }^{(12)}$.

Existen otras formas de alteración de flujo en arterias abdominales como es el caso del síndrome de compresión de tronco celiaco ${ }^{(13)}$, en el cual se comprime el origen de éste por el ligamento Arcuato del diafragma, por donde atraviesa la aorta del tórax al abdomen. Esta compresión se exacerba a la inspiración profunda que es cuando el diafragma desciende y el ligamento comprime el tronco celiaco hacia la aorta.

De manera primaria, las indicaciones para intervenir en una isquemia mesentérica, ya sea con cirugía abierta o endovascular son: signos de infarto intestinal, signos de ruptura arterial, dolor abdominal persistente a pesar del tratamiento, compresión severa u oclusión de la luz verdadera y dilatación aneurismática de la AMS ${ }^{(9)}$.

Cuando ya se decide intervenir, hay que dilucidar entre el manejo endovascular y la cirugía abierta; y un punto de quiebre es la presencia de peritonismo, signo que aboga más por la cirugía abierta. De no haber peritonismo, la tendencia es el intervencionismo. En intervencionismo se puede realizar procedimientos mecánicos o farmacológicos, instilando en estos últimos, drogas líticas, anticoagulantes o vasodilatadores, entre otros. Los mecánicos pueden ser dilataciones con balón, balón con drogas, colocación de stents, trombectomía fármaco-mecánica, etc. Entre los procedimientos abiertos se cuenta la trombectomía, la endarterectomía, y el by pass de vena o de injertos artificiales.

En los casos de isquemia mesentérica arterial aguda se debe considerar la cirugía abierta o endovascular antes de la cirugía intestinal; con recomendación clase II-a, y nivel de evidencia $\mathrm{B}^{(10)}$. Asimismo, en casos de oclusión trombótica aguda de la AMS, debe considerarse la terapia endovascular como primera línea debido a menor mortalidad y menor resección intestinal, comparado con la cirugía abierta: Recomendación II-a y nivel de evidencia B ${ }^{(10)}$. En los casos de isquemia venosa, el manejo es anticoagulación, predominantemente.

La elección de procedimientos endovasculares en los cuatro casos presentados fue por la premura (tiempo) por aminorar el dolor y el control de daño; además de ser un buen tratamiento en los casos de disección y trombosis. El no presentar signos de peritonismo o sufrimiento grave intestinal abogó más aún para el abordaje intervencionista. En el caso cuatro, a quien se le hizo una colecistostomía de inicio, debido a un estado general deteriorado, fue una razón más que suficiente para realizar una intervención endovascular en contraparte a una cirugía abierta.

Los procedimientos endovasculares son cada vez más frecuentes en los vasos viscerales y se realizan cada vez con mayor familiaridad. Los resultados a corto y mediano plazo del intervencionismo visceral son adecuados, acortan el inicio del tratamiento y reducen la posibilidad de resección intestinal si son indicados antes de llegar a estadios avanzados ${ }^{(6)}$, especialmente en la isquemia aguda o reagudizada. Son, además, procedimientos factibles y reproducibles. En nuestros casos la evolución ha sido bastante adecuada, con las limitaciones de ser una muestra pequeña, con un seguimiento corto al momento de relatar este registro. Se requiere un armamentario endovascular refinado tecnológicamente y una infraestructura de costos elevados pero con una relación costo-beneficio siempre favorable.

\section{Contribución de autoría:}

HAS: Redacción, revisión de la literatura y aprobación de la versión a ser publicada; PCM: redacción del artículo y revisión de la literatura; NDS y JCdHC: Revisión de la literatura; LBO y ATA: contribución en el manejo asistencial.

\section{Correspondencia:}

Hernán Aste S.

Clínica SANNA - San Borja

Av. Guardia Civil 337, San Borja.

Lima, Perú.

Correo Electrónico: hernan.aste@sanna.pe / haste@

cardiotrainingperu.com

Móvil:+51 999706976 


\section{REFERENCIAS BIBLIOGRÁFICAS}

1. Motta GA, Sánchez-García JC, Ontiveros-Rodríguez A, et al. Isquemia Mesentérica Aguda: Urgencia que exige un abordaje diagnóstico integral. An Radiol Méx. 2015; 14:66-88.

2. Lee RW, Bakken AM, Palchik E, et al. LongTerm Outcomes of Endoluminal Therapy for chronic atherosclerosis occlusive mesenteric disease. Ann Vasc Surg. 2008; 22:541-6.

3. Sun J, Li DL, Wu Z, He Y, Zhu Q, Zhang H. Morphologic findings and management strategy of spontaneous isolated dissection of the celiac artery. $\mathrm{J}$ Vasc Surg. 2016; 64:389-94.

4. Yun WS, Kim YW, Park KB, et al. Clinical and angiographic follow-up of spontaneous isolated superior mesenteric artery dissection. Eur J Vasc Endovasc Surg. 2009; 37:572-7.

5. Luan JY, Li X. Computed tomography imaging features and classification of isolated dissection of the superior mesenteric artery. Eur J Vasc Endovasc Surg 2013; 46(2):232-5.

6. Cronenwett JL, Wayne Johnston K. Rutherford's Vascular Surgery. Philadelphia, PA. USA: Elsevier Saunders; 2014. p. 2360-420.

7. Talledo O, Torres L, Valenzuela $\mathrm{H}$, et al. Dolor abdominal persistente por disección de arteria mesentérica superior y tronco celiaco, que no responden a tratamiento conservador. Rev Gastroenterol Peru. 2017; 7(3):262-6.
8. Morgan CE, Mansukhani NA, Eskandari MK, Rodriguez HE. Ten year review of isolated spontaneous mesenteric arterial dissections. J Vasc Surg. 2018; 67:1134-42.

9. Li DL, He YY, Alkalei AM, et al. Management strategy for spontaneous isolated dissection of the superior mesenteric artery based on morphologic classification. J Vasc Surg. 2014; 59:165-72.

10. Björck M, Koelemay M, Acosta S, et al. Management of the Diseases of Mesenteric Arteries and Veins: Clinical Practice Guidelines of the European Society of Vascular Surgery (ESVS). Eur J Vasc Endovasc Surg. 2017; 53:460-510.

11. Oderich GS, Tallarita T, Gloviczki P, et al. Mesenteric artery complications during angioplasty and stent placement for atherosclerotic chronic mesenteric ischemia. J Vasc Surg. 2012; 55:1063-71.

12. Oderich GS, Erdoes LS, LeSar C, et al. Comparison of covered stents versus bare metal stents for treatment of chronic atherosclerotic mesenteric arterial disease. J Vasc Surg. 2013; 58:1316-24.

13. Van Petersen AS, Kolkman JJ, Gerrits DG, et al. Clinical significance of mesenteric arterial collateral circulation in patients with celiac artery compression syndrome. J Vasc Surg. 2017; 65(5):1366-1374. doi: 10.1016/j.jvs.2016.11.052.

Recibido: $22 / 06 / 2020$ Aceptado: 30/03/2021 